\title{
Suicide among the Elderly: A Psychological Perspective
}

\author{
Shatabdi Das \\ Department of Psychology, Osmania University College for Women, Koti, Hyderabad, Telangana State -500095, India
}

\begin{abstract}
This theoretical paper focusses on the psychological aspects of understanding the impact of elderly suicide on society. The reasons associated with the prevalence of suicide among the elderly population and the preventive measures that can be utilised for enhancement and adjustment of the elderly with respect to their standing in their personal and social life. The elderly population of India has a noteworthy risk of suicide compared to the other age groups. Hence, it becomes pertinent to identify the suicidal risk factors prevalent among the elderly, which can further help in planning and prevention of suicides among this group. It has been found that amongst the Indian geriatric populace factors such as, co-morbid physical illness, mental illness, and family burden of psychiatric illness, lower educational background, unemployment, widowhood are prominent predictors for elderly suicide attempts. Thus, suicide prevention for the elderly needs specific interventions specially personalised for this population, such as, familial and social support, improved identification, assessment and treatment of depression, attention to medical problems, dependency issues, and education of primary care physicians can be promoted as interventions for reduction of suicide among the elderly.
\end{abstract}

Keywords Indian Population, Elderly, Suicide, Geriatric, Prevention Strategies, Old Age

\section{Introduction}

The word "Suicide" has been derived from the Latin word called "suicidium", which means 'to kill oneself'. It has been found to be one of the leading causes of death among elderly people (here, elderly is considered to be a person with age of 65 or plus years). Various studies related to finding the rate of suicide among the elderly have shown that in several countries the rate of suicides among elderly people are higher than or is as high as that of young people. Even after these substantial findings, still study on suicide in old age is a neglected area. As a matter of fact, there is a lack of even basic knowledge about elderly suicide among clinicians. WHO estimated the suicide rate for men and women aged 75 and above to be 50 for 100,000 and 16 for 100,000 respectively (Heisel, 2005). In a study from South India (Hall, Platt \&, Hall, 1999) it was found that the suicide rate in the population above 55 years of age is 189 for 100,000 . Around one in five of all successful suicides are committed by individuals above the age of 65 years. Some prognosticators of suicide in old age have been described in studies, such as mental health, personality, physical diseases, social factors (Conwell \& Thompson, 2008), psychiatric disorders (Waern, Rubenowitz, Runeson, Skoog, Wilhelmson \& Allebeck, 2002) (particularly depression), physical illness, functional impairment, and stressful life events increases the risk for suicide in the elderly (Erlangsen, Jeune, Bille-Brahe \& Vaupel, 2004).

Surveys from the last two decades, indicates that the suicide rate has increased from 7.9 to 10.3 per 100,000 , (Vanderhorst \& McLaren, 2005) with very high rates in some southern states. In the surveys of the general population (Joseph, Abraham, Muliyil, George, Prasad, Minz, Abraham \& Jacob, 1994) it was established that age and gender specific suicide rates (Aaron, Joseph, Abraham, Muliyil, George \& Prasad, 2004) and was found to be 234 in 100,000 and 147 in 100,000 among elderly men and women, respectively (Abraham, Abraham \& Jacob 2005).

As the proportion of the elderly age group in the population will increase all over the world in the coming decades, so is the number of suicides among older adults is expected to be on the rise consequently. Therefore, proper identification of elderly at risk is important for early intervention in consideration with the policies of the public health maintenance.

It has been found that elderly individuals usually consult primary health care providers' more than mental health professionals for their mental health related issues. So, knowledge regarding the suicidal risk factors specific to elderly population could be considered as a first step in the formulation of suitable interventions for suicide prevention in the elderly in keeping with reference to the Indian context.

It has been noticed that there has been a global trend toward increased rate of suicide in late life, especially among men. (Crepet, Caracciolo, Casoli, Fabbri, Florenzano \& Grassi, 1991 \& Adamek, Kaplan, 1996). Though, in a longitudinal study spanning over 5 years, out of 6312 suicide 
attempters, only 47 were above the age of 60 years. This low occurrence of suicide among the elderly in India can be attributed to the well-integration and respect of the elderly in the family, as younger ones take responsibility and care for them (Rao \& Madhavan, 1983). Another reason to be considered is that the, life expectancy of the elderly is lower in India than elsewhere, which can be contributing to the comparatively lower suicide rate (Shukla, Verma \& Mishra, 1990).

In a contrasting study however, it was found that the ratio of completed suicide to attempted suicide in India is about 1:7 in the elderly, which is found to be that of double the ratio of 1:15 in lower age groups. This may determine the poor ability of the elderly to recuperate from the bodily insult of a suicide attempt (Rao \& Madhavan, 1983). Studies from the west associate social isolation, as defined by 'living along' as a risk factor for suicide among the elderly. But another study assumed that for elderly in India, family and social integration were the significant factors of risk, even if they were living by themselves (Rao, 1991 \& Purcell, Heisel, Speice, Franus, Conwell \& Duberstein, 2011).

\section{Factors Influencing Suicidal Tendencies among the Elderly}

\section{Demographical Factors}

Education has been found to be a determinant, as most of the elderly attempters are found to be less educated than their younger counterparts. The reason may be attributed to the proportional lack of emphasis on education and dearth of resources in previous decades.

Unemployment is another determinant of suicide in the elderly attempters as comprehensibly many of them usually would be retired. Unemployment leading to lack of steady income and hence dependence on others for economic support are reported to be a risk factor for suicide in the elderly.

There had been prevalence of suicide attempts from the rural areas (considering the catchment area of the hospital), in comparison to urban areas. This may be due to the fact that the elderly are left behind in rural areas making them vulnerable to social isolation. And social isolation is considered as a causative factor for the rising rate of suicide with the increase in age.

The family backgrounds have also been found to be the causative factors for the suicide attempters. And elderly from joint family background are found to be more susceptible to suicidal bents than elderly belonging to extended family backgrounds. The reason is likely to be that even though these elderly stay in joint family settings, still they may not be getting the adequate emotional and physical support that they require for their sustenance from their family members.

\section{Physical Comorbidities}

There has been found to be a substantial relationship between physical illness and suicide in the elderly ages. Many studies have found a connotation between poor physical health and suicide. There has been link found between physical illness and suicide which is further mediated by depression and a sense of hopelessness which, again, is prominently more common in elderly (Conwell, Lyness, Duberstein, Cox, Seidlitz \& Di Giorgio, 2000). As physical illnesses generates stress in life, and rises the burden on care givers, that can further cause family discordance and hence can drain the financial resources. Physical illness also deprives one's independent behaviour which can cause an unrepairable dent in one's self-esteem, in a society that places importance on autonomy.

In significant studies it have been found that elderly people with suicidal inclinations recurrently refer to medical services, generally a primary health care physician, mostly before the suicidal attempt, sometimes in the last few days or hours preceding the suicidal attempt (Luoma, Martin \& Pearson, 2002). Which provides an opportunity for the health care experts to prevent the elderly from taking the drastic step of committing suicide.

In a population based case-control study, it was found that factors such as, visual impairment, neurological disorders, malignant disease, cardiovascular disease, and musculoskeletal disorders were related with suicide in elderly people (Waern et al., 2002).

\section{Psychiatric Illness}

Unipolar depression has been found to be the most common Axis-I diagnosis associated with suicide among the elderly people (Axis-I is part of the Diagnostic and Statistical Manual of Mental Disorders, DSM-IV which is organized into a five-part multiaxial system for assessment of all psychologically diagnostic categories except for mental retardation and personality disorders).Many general practitioner fail to determine the indicative signs of depression in the elderly and attribute it to usual complaints associated with aging. This lack of recognition is aggravated by the fact that the depression often presents in a different way in the elderly which is often confused with other concomitant medical conditions. Elderly persons suffering from depression usually show fewer mood symptoms and instead complain of fatigue, lack of concentration, weakened memory, and lack of initiative. Frequently, establishing the diagnosis and initiating treatment in elderly is difficult as the diagnostic systems require a significant number of symptoms to make the diagnosis of depressive disorder. The problem is further magnified by the lack of sensitization of the primary health care givers with respect to the geriatric mental health issues. 
It has also been found that elderly group has higher rates of past psychiatric illness and family history of psychiatric illness in first degree relatives than the younger age group. This proposes that the most suicide attempters in the elderly group suffer from co-morbid psychiatric disorder compared to the young age group where attempts are more likely to be related to immediate life events.

In comparison to the younger attempters where majority attempts suicide within 24 hour after a stressor, the majority of the elderly attempters however may attempt suicide more than a week after a stressor has occurred. This suggests that the suicide attempts in the elderly group are 'premeditated and less impulsive'. This yet again ties up with the fact that elderly suicides appear to be related more with mental and physical illnesses and related worries about dependency.

Older people may also attempt suicide due to intolerable psychological pains, which produces an amplified state of disconcertion. The person may want firstly to flee away from pain, by feeling boxed up, rejected, feelings of hopelessness and helpless (Leenaars, 2003). The suicide is serviceable because it provides relief from the unbearable suffering. A history of suicide attempts along with the level of intent associated with suicidal attempts has been found to be correlated with the succeeding completed suicides (Connor, Conwell \& Duberstein, 2001).

Incompetence to adjust with disorders is another motive for older adult to attempt suicide. This comprises several disorders such as depressive disorders, anxiety disorders, schizophrenic disorders, brain-dysfunction disorders, and substance-related disorders.

The various reasons for older adults to attempt suicide are, rejection-aggression, it was first documented by Stekel in the 1910 meeting of the Psychoanalytic Society in Vienna (Leenaars, 2003). The idea behind this reason is that often at times of rejection it leads to pain and self-directed aggression.

Identification-aggression, is an idea hypothesized by Freud, is another factor in older adult suicidal tendencies. With this idea, Freud believed that intense identification or attachment with a lost or rejecting person is critical in understanding the suicidal person's intensions. If this emotional attachment is not met, the suicidal person experiences a deep discomfort and wants to escape it through suicide (Leenaars, 2003).

Interpersonal relationships are regularly a factor in older adult suicides. If older adults have trouble establishing or maintaining relationships, they develop disturbed, insufferable interpersonal tragedies.

Cognitive constriction is another factor in older adult suicide. The common cognitive state in suicide is mental constriction, such as rigidity in thinking, narrowing of focus, tunnel vision, and concreteness (Leenaars, 2003). The person experiences amalgamations of trauma such as poor health or rejection from a family member, moments before his or her death.

Indirect expressions are a reason for suicide among older adults. The suicidal person is indecisive, they experience complications, conflicting feelings, attitudes or drives, often towards a person and even towards life (Leenaars 2003). However, the conscience of a person is only a fragment of the suicidal mind (Leenaars 2003).

\section{Mental Health}

Conwell, Duberstein, Cox, Herrmann, Forbes \& Caine (1996), found in an assessments of late life suicide, that from $71 \%$ to $95 \%$ of suicide victims age 65 years and older had a major psychiatric disorder, often, major depression, at the time of death. Primary psychotic illnesses, such as, schizophrenia, schizoaffective disorder, and delusional disorder, personality disorders, and anxiety disorders were found to attribute a relatively small role in suicide among older adults.

In distinction, elderly suicide victims are more likely to have suffered from depressive illness than their younger counterparts (Conwell \& Brent, 1995). In a PA (PA is Psychological Autopsy method, is an attempt to determine any emotional or psychological factors which might have caused to an individual's suicide) study of 141 completed suicides, demonstrated that greater age at death was significantly associated with a diagnosis of single episode, unipolar major depression (Conwell et al., 1996). The clinical depression was of moderate severity and irregularly associated with comorbid substance abuse disorders, suggesting a likelihood of responses to standard therapies (Conwell, Duberstein \& Caine 2002). Relative to its frequency in older adults, dementia is infrequently diagnosed in completed suicides in younger individuals, as determined by the PA method. Overall, these studies suggest that affective illness is the predominant psychopathology associated with suicide in late life (Conwell et. al., 2002).

Results of the potential, non-clinical cohort study of suicide in older adults completed proposes that in addition to being widowed or divorced, the strongest predictor of suicide was self-rated depression symptom severity (Ross, Bernstein, Trent, Henderson \& Paganini-Hill, 1990).

A five PA studies of suicide in late life have encompassed comparison samples with which to establish base rates of presumed risk factors, and thus, the relative risk related with each factor (Conwell et al., 2002). In these studies, the presence of an Axis I mood disorder was evidently and strongly (an odds ratios ranging from 27.4 to 113.1) associated with raised risk for suicide in older adults. Recurring major depression was associated with the greatest risk, yet single episode of major depression, dysthymia, and minor depression were also significant interpreters of completed suicide. Three of four PA studies examined individuals with a diagnosis of dementia and found no significant difference between suicidal subjects and controls. Psychotic disorders were predictive of suicide in only one of five studies. The results for substance abuse disorders were however mixed, with three of five studies showing a 
statistically significant rise of risk. And all three studies that examined a prior history of suicide attempts found it to be a statistically significant risk factor (Conwell et al., 2002).

Two studies that included standardized measures of personality traits (Duberstein, Conwell \& Caine, 1994) established that suicide victims over the age of 50 years were distinguished from age-matched controls by higher levels of Neuroticism $(\mathrm{N})$ and lower scores on the Openness to Experience (OTE) factor on Costa and McCrae's NEO Personality Inventory (Costa \& McCrae, 1992). Low OTE describes individuals with muted affective and hedonic responses, a limited range of interests, and comfort with the familiar. The researchers hypothesized that suicide risk is increased in older persons with low OTE because of their limited adaptability to the challenges of aging and because their distress which may be more difficult for others to perceive. Harwood and associates compared suicides and natural death controls for age 60 years and over on ICD-10 personality trait accentuation (Harwood, Hawton, Hope \& Jacoby, 2001). Anankastic (obsessional) and anxious traits, which the authors note were qualitatively similar to low OTE scores, significantly distinguished by the groups (Harwood, Hawton, Hope \& Jacoby 2001).

\section{Social Attributions}

Daily hassles keep on gathering through times which may lead to stress further leading to suicide attempts in older adults (Luscomb, Clum \& Patsiokas, 1980). The explicit types of life events most relevant to suicide in late life diverge from those of other age group sufferers. It has been found that among the young and middle adulthood the more typical reasons for suicidal tendencies are interpersonal discordance, financial or job difficulties, and legal difficulties, in contrast the most typical stressors in older adults attempting for suicide are physical illness and emotional losses (Carney, Rich, Burke \& Fowler, 1994; Conwell, Rotenberger \& Caine, 1990; Heikkinen \& Lonnqvist, 1995). Studies of suicide victims with census data have found that older adults who commit suicide were more likely in the community to have lived alone in comparison to other older adults who have not experienced living alone or without family or friends, this signifies that social isolation and loneliness are vital factors to be considered (Barraclough, 1971). A relevant circumstance may be the complex construct of social support and the moderating role that it may play in determining the risk for suicide associated, such as, with stressful life events like bereavement, or unusual, but conspicuous cases of homicide, or suicide in older adults proposes that risk can also be related with caregiver affliction as well (Cohen, Llorente \& Eisdorfer, 1998).

Studies using the PA method have compared the magnitudes of individuals who completed suicides and controls cases of those who lived alone, (Beautrais, 2002; Conwell, 2001; Conwell, Lyness, Duberstein, Cox, Seidlitz $\&$ Di Giorgio, 2000) and one study with this proposition found significant difference between the groups (Conwell, 2001). On the similar line of concern, two studies with specific stressors in cases and controls, that is, financial and relationship problems (Beautrais, 2002) and family discord (Rubenowitz, Waern, Wilhelmsson \& Allebeck, 2001) distinguished the groups. And, it was noted that when depressive symptoms were statistically controlled for, then only the family discord remained predictive for late life suicide attempts (Conwell et al., 2002).

Researches considering social support as a cause are more constant across studies than findings regarding living situations and stressful life events. Miller (1978), testified that controls were significantly more likely than suicide victims to have had a confidant and to have good relationships with friends or relatives. Conwell, et.al (2000), found that elderly controls had received significantly greater support with their daily tasks and had greater social contact than elderly suicide completers who had no or far less support in their completions of daily tasks. Beautrais, (2002) found that low social interaction was also a substantial risk factor for suicide even after adjusting the physical and mental health variables in a sample studied from New Zealand. Hence, life events and social supports appear to establish risk factors to suicide in later life. However, it is indistinct to the extent to which their effects may be arbitrated by associations with other variables, such as depression. Further, the probable roles of mediators such as personality and culture warrant a more sustained investigation.

In a research done by Iris Chi, DSW, and Golden Age Association Frances Wu, Chair for Chinese Elderly School of Social Work \& Davis School of Gerontology at the University of Southern California in Los Angeles, found that elderly people with personality types that are less open and are of more inward nature display a greater susceptibility towards committing suicide.

\section{Detection of the Warning Signals of Suicide}

Family members and experts should be attentive to any change in behaviour among the older adults that can help to determine their suicidal tendency.

Many people in the society consider depression to be the main sign for detecting suicidal tendencies among the elderly, but other factors, such as, sleep problems (either too much or too little sleeping), eating problems, should also be taken into consideration as the signs of suicidal tendencies.

Various factors, such as, attitudes, beliefs like, autonomy, dignity, and responsibility can also be significant attributors in suicidal tendencies.

Additionally, habits, such as, alcohol and other substance abuse disorders also projects elderly people at augmented risk for suicide.

Possession of any weapon or firearms in the household has been found to positively increase the risk. 
Verbal statements of wanting to die or kill oneself should be taken seriously and professional help should be sought for.

Thus, every community should have mental health professionals as well as helplines or even suicide prevention centres to which the elderly in distress can turn for help and support.

\section{Prevention of Suicide among the Elderly}

The efficiency of any method designed for prevention of suicide among the elderly depends largely upon the extent to which the causal factors have been recognised, the strengths of the causal relationships to suicide, their frequency in the elderly population, and the extent to which they can be altered (Somers, 1985). Research data indicates that factors such as affective illness, past history of suicidal behaviour, hopelessness, and physical syndromes along with functional deficiencies should be emphasised while considering for future preventive efforts (Conwell, 1997).

Primary identification and suitable treatment of a crucial psychiatric disorder is of utmost importance. For psychiatrically sick individuals, several antipsychotic drugs, antidepressants, and behavioural interventions such as dialectical behavior therapy, (Dialectical behavior therapy $(D B T)$ is a specific type of cognitive-behavioural psychotherapy), used for the treatment of various kinds of mental health disorders have been found $t$ to have anti-suicidal effects.

As the most prevalent predictor of completed suicide is the presence of a previous suicide attempt, interventions then should be aimed at suicide attempters, which may be the most effective in decreasing suicide rates. Vijayakumar, Umamaheswari, Shujaath, Devaraj, Kesavan (2011), examined the effectiveness of short-term intervention and regular contact in a randomized controlled trial in suicide attempters and found that it reduced the rates of completed suicide over a following 18-month period. Significantly though, the care received by the treatment-as-usual arm in this study was below the needed standards because it was limited to the critical management of the somatic sequelae of the suicide attempt and it did not include any psychiatric or psychological assessment or treatment.

In considering the psychological autopsy report, it was found that $24 \%$ of suicide completers had consulted a psychiatrist or a physician before committing suicide, and in $68 \%$ of cases the family of the victim were aware of their suicidal intent. This finding is comparable to data from the west, where two-third of the elderly suicide committers visited their general practitioners in the month prior to death, and $40 \%$ did the same in the week before. This appeals for adequate training of general practitioners in primary detection and referral of patients with common mental disorders, which may help in significant waning in suicide rates. But it also have to be culturally sensitive; as the higher rate of somatic symptoms, rather than cognitive symptoms, among depressed patients in the Indian setting, is a case in point.

The primary identification and treatment of vulnerable populations with risk factors for suicide across the life-span can be used as another strategy to prevent the elders from committing or attempting of suicide. Further at the community level, the conduction of social programs such as individual and family support programs and programs aimed at achieving sensitisation towards the elderly issues and concerns may prove to be useful.

The need for a strategy which will raise awareness and help make suicide prevention a national priority has long been acknowledged. Such a national strategy will need a comprehensive approach that encompasses the promotion, coordination, and support of activities to be executed across the country at national, regional, and local levels. The program would need to be personalised for populations at risk. Strategies with empirical evidence in western literature such as the Universal, Selective and Indicated model (USI), 'gatekeeper training', outpatient follow-up and emergency outreach may also be applicable in Indian context. The USI model outlines 'universal' preventive strategies for the population as a whole (e.g., restricted access to lethal means), 'selective' strategies targeting at-risk individuals (e.g. psychiatrically ill, homeless, socially-excluded groups) and 'indicated prevention' strategies aiming at suicide attempters (e.g. outpatient contact and emergency outreach). Gatekeeper training focuses on skill development to enable community members such as teachers, coaches and others in the community to identify signs of depression and suicide-related behaviors among various age groups. It encourages individuals to maintain a high index of distrust and to inquire directly about distress, convince suicidal individuals to accept help, and serve as a link for local referrals. Such approaches would also require a multidisciplinary team approach involving psychiatrists, general physicians, psychiatric nurses, psychiatric social workers, and non-governmental organizations (NGOs).

The role of the media has also become progressively relevant. A delicate balance needs to be maintained between press freedom and responsibility of the press to minimize the harm to susceptible individuals. The role of advocacy and legislature cannot be over-emphasized. Laws restricting availability of lethal agents such as firearms have been advocated by the WHO.

Taking action to help can include getting the word out (that someone is in danger of committing suicide) into the stream of communication, letting others know about it, breaking what could be called a fatal secret, talking to the person directly, talking to others, offering help, getting family members interested and responsive, creating action around the person, showing response, and, if possible, showing genuine and deep concern.

Options for prevention can contain various strategies, including limiting access to firearms and reducing the 
inappropriate use of sedative medications. Most prominently, educational programs tailored for primary health care providers on the identification and treatment of late-life depression can be a vital component of lowering suicide rates. Evidence shows that most elderly suicide victims visit their physician shortly before dying. In fact, over $70 \%$ of older patients who die by suicide visit their primary care physician within a month of their death.

Problems with public notions of what is and is not acceptable with regard to suicide and the belief that old age equals depression, contributes to the lack of appropriate treatment of suicidal behavior and disorders among the elderly. The family can contribute to the risk--or to the reduction--and treatment of suicidal older adults.

A qualified mental health practitioner, such as a family therapist, who is experienced with handling issues of the elderly, including depression and high risk for suicide, can provide services to help the suffering older adult, as well as assist the family as they cope with various issues surrounding the problem.

Over $80 \%$ of geriatric patients in one study recovered from depression when treated with brief therapy combined with medication for depressive disorders.

Elderly suicide will continue to be a major public health problem as the baby boomer (baby boomer is a person born in the years following the Second World War, when there was a momentary marked increase in the birth rate) generation enters retirement. More efforts surrounding community-based care, mental health, funding and legislative initiatives must be focused on this age group to reduce this preventable tragedy at the end of life.

Association with multiple factors in geriatric suicide attempters permits broadly directed and multimodal interventions. Enhanced identification of depression, better treatment of depression, adequate attention to medical problems and associated dependency issues, and sensitization and education of primary care physicians could be the targeted interventions for reduction of suicide prevalence in old age. As suicide in the elderly needs specific interventions tailored for this population rather than a nonspecific, "one size fits all" approach.

Hence, the approach to understand suicide must be multidisciplinary, involving psychologists, psychiatrists, toxicologists, physiologists and physicians, as suicide is a multi-faceted and complex event. The premature and violent death of the victims has negative repercussions in society and should be prevented whenever possible.

\section{Acknowledgements}

My sincere gratitude to the anonymous reviewers, Ms. Elizabeth Carter, Dr. Venkat Pulla \& Mr. Kevin Nelson, for giving me valuable inputs and accepting my paper for the publication in the reputed journal.

I would also like to forward my heartfelt gratitude to Dr. Vinita Pandey for providing me the guidance and help as a peer mentor.

\section{REFERENCES}

Aaron R, Joseph A, Abraham S, Muliyil J, George K, Prasad J, et al. Suicides in young people in rural southern India. Lancet; 363:11178, 2004.

Abraham VJ, Abraham S, Jacob KS, Suicide in the elderly in Kaniyambadi block, Tamil Nadu, south India. Int J Geriatric Psychiatry; 20:953-5, 2005.

Adamek. M.E, Kaplan. M.S, Firearm Suicide among Older men, Psychiatric Services, 47(3):304-6, 1996.

Barraclough. B.M, Suicide in the elderly: Recent developments in psychogeriatric, British Journal of Psychiatry, Suppl 6:87-97, 1971.

Beautrais. A.L. A case control study of suicide and attempted suicide in older adults, Suicide \& Life Threatening Behavior. 32 (1):1-9, 2002.

Chi. I, Cross-cultural gerontology research methods: Challenge and solution, In Methodologies for Diverse Ageing Population, (in press)

Conwell. Y, Rotenberg. M, Caine .E.D, Completed suicide at age 50 and over, Journal of the American Geriatric Society, 38(6):640644, 1990.

Conwell. Y, Brent. D, Suicide and aging. I: Patterns of psychiatric diagnosis, International Psychogeriatric, 7(2):149-164, 1995.

Conwell. Y, Duberstein. P.R, Cox. C, Herrmann. J.H, Forbes. N, Caine. E.D, Relationships of age and Axis I diagnoses in victims of completed suicide: A psychological autopsy study, American Journal of Psychiatry, 153(8):1001-1008, 1996.

Conwell .Y, Management of suicidal behavior in the elderly, Psychiatric Clinics of North America, 20 (3):667-683, 1997.

Cohen. D, Llorente. M, Eisdorfer. C, Homicide-suicide in older persons, American Journal of Psychiatry, 155(3):390-396, 1998.

Connor. K., Conwell. Y, Duberstein. P, The validity of proxy-based data in suicide research: A study of patients 50 years of age and older who attempted suicide. II, Life events, social support and suicidal behavior, Acta Psychiatrica Scandinavia, 104, 452-457, 2001.

Conwell. Y, Lyness. J.M, Duberstein. P, Cox. C, Seidlitz. L, Di Giorgio. A, et al, Completed suicide among older patients in primary care practices: A controlled study, J Am Geriatric Society, 48:23-9, 2000.

Conwell. Y, Suicide in Later Life: A Review and Recommendations for Prevention, American Association of Suicidology, Vol. 31, Iss.1, 32-47, 2001.

Conwell. Y, Duberstein. P.R, Caine. E.D, Risk factors for suicide in later life, Biological Psychiatry, 52(3):193-204, 2002.

Conwell. Y, Thompson. C, Suicidal behavior in elders, Psychiatric Clinic North America, 31: 333-56, 2008.

Costa. P.T, McCrae. R.R, Revised NEO Personality Inventory and NEO Five Factor Inventory: Professional manual, Odessa, FL: PAR; 1992. 
Crepet. P, Caracciolo. S, Casoli. R, Fabbri. D, Florenzano. F, Grassi .G.M, et al., Suicidal behavior in Italy: Data, trends and guidelines for a suicide intervention/prevention policy, Suicide Life Threat Behaviour, 21:263-78, 1991.

Derasari. S, Shah. V.D, Comparison of symptomatology of depression between India and U.S.A, Indian J Psychiatry, 30:12934, 1998

Duberstein. P.R, Conwell. Y, Caine. E.D, Age differences in the personality characteristics of suicide completers: Preliminary findings from a psychological autopsy study, Psychiatry, 57(3):213-224, 1994

Duberstein. P.R, Conwell. Y, Seidlitz. L, Lyness. J.M, Cox. C, Caine .E.D, Age and suicidal ideation in older depressed inpatients, Am J Geriatric Psychiatry, 7: 289-96, 1999.

Erlangsen A, Jeune B, Bille-Brahe U, Vaupel JW. Loss of partner and suicide risks among oldest old: A population-based register study. Age Ageing; 33:378-83, 2004.

Hall R.C, Platt D.E \& Hall R.C, Suicide risk assessment: A review of risk factors for suicide in 100 patients who made severe suicide attempts. Evaluation of suicide risk in a time of managed care. Psychosomatics; 40:18-27, 1999.

Harwood D, Hawton K, Hope T, Jacoby R, Psychiatric disorder and personality factors associated with suicide in older people, International Journal of Geriatric Psychiatry, 16(2):155-165,2001.

Heisel M, Duberstein PR. Suicide prevention in older adults. Sci Pract. 2005; 12: 242-59.

Houston. K, Haw. C, Townsend. E, Hawton. K, General practitioner contacts with patients before and after deliberate self-harm, Br J Gen Pract, 53:365-70, 2003.

Isaac. M, Elias. B, Katz. L.Y, Belik. S.L, Deane. F.P, Enns. M.W, et al., Gatekeeper training as a preventative intervention for suicide: A systematic review, Can J Psychiatry, 54:260-8, 2009.

Joseph. A, Abraham. S, Muliyil. J. P, George. K, Prasad. J, Minz. S, Abraham. V. J. \& Jacob. K.S, Evaluation of suicide rates in rural India using verbal autopsies, 1994-9, The British Medical Journal, 326(7399): 1121-1122, 2003

Leenaars. A, Can a theory of suicide predict all "suicides" in older adults? Crisis, 24, 7-16, 2003.

Linehan. M.M, Comtois. KA, Murray .A M, Brown. M.Z, Gallop. R.J, Heard. H.L, et al., Two-year randomized controlled trial and follow-up of dialectical behavior therapy vs therapy by experts for suicidal behaviors and borderline personality disorder, Arch Gen Psychiatry, 63:757-66, 2006.

Luoma. J.B, Martin. C.E, Pearson. J.L, Contact with mental health and primary care providers before suicide: A review of the evidence. Am J Psychiatry, 159:909-16, 2002.

Luscombe. R.L, Clum. G.A, Patsiokas. A.T, Mediating factors in the relationship between life stress and suicide attempting, Journal of Mental Disorders, 168(11):644-650, 1980.

Manoranjitham. S, Abraham. A, Jacob. K.S, Towards a national policy to reduce suicide in India, Natl Med J India, 18:118-22, 2005.

Miller. M, Geriatric suicide: The Arizona study, Gerontologist, 18(5 Pt 1): 488-495, 1978 .

Nordentoft M. Prevention of suicide and attempted suicide in
Denmark. Epidemiological studies of suicide and intervention studies in selected risk groups. Dan Med Bull. 2007; 54: 306-69.

Prichard \& Amanullah, Trends in rates and methods of suicide in India, Egyptian Journal of Social Sciences, Vol. 3, Issue. 3, 2007.

Purcell. B, Heisel. M.J, Speice. J, Franus. N, Conwell .Y, Duberstein. P.R, Family connectedness moderates the association between living alone and suicide ideation in a clinical sample of adults 50 years and older, Am J Geriatric Psychiatry, 2011.

Rao. A.V, Madhavan. T, Depression and suicide behaviour in the aged, Indian J Psychiatry, 25:251-9, 1983.

Rao. A.V, Suicide in the elderly: A report from India, Crisis, 12:339, 1991.

Ross. R.K, Bernstein. L, Trent. L, Henderson. B.E, Paganini-Hill A, A prospective study of risk factors for traumatic death in the retirement community, Preventive Medicine, 19(3):323-334, 1990.

Rabinowitz. E, Waern. M, Wilhelmson. K, Allebeck. P, Life events and psychosocial factors in elderly suicides: A case control study, Psychological Medicine, 31(7):1193-1202, 2001.

Rutz. W, Von Knorring. L, Walinder. J, Frequency of suicide on Gotland after systematic postgraduate education of general practitioners, Acta Psychiatric Scand, 80: 151-4, 1989.

Shah. A, The relationship between suicide rates and age: An analysis of multinational data from the World Health Organization, Int Psychogeriatric, 19: 1141-52, 2007

Sharma. B.R, Gupta. M, Sharma. A.K, Sharma. S, Gupta. N, Relhan .N, et al. Suicides in northern India: Comparison of trends and review of literature. J Forensic Leg Med. 2007; 14:318-26.

Shukla. G.D, Verma. B.L, Mishra .D.N, Suicide in Jhansi city, Indian J Psychiatry, 32:44-51, 1990.

Somers .A.R, Preventive health services for the elderly. In: Andres R, Bierman EL, Hazzard WR, editors, Principles of geriatric medicine and Gerontology, New York: McGraw Hill, pp. 120-125, 1985.

Soomro. G.M, Deliberate self-harm (and attempted suicide) Clin Evid (Online) 2008.

Turvey. C.L, Conwell. Y, Jones. M.P, Phillips. C, Simonsick. E, Pearson. J.L, et al., Risk factors for late-life suicide: A prospective, community-based study, Am J Geriatric Psychiatry, 10:398-406, 2002 .

Vanderhorst. R.K, McLaren. S, Social relationships as predictors of depression and suicidal ideation in older adults, Aging Mental Health, 9:517-25, 2005.

Vijayakumar. L. Suicide prevention: The urgent need in developing countries, World Psychiatry, 3:158-9, 2004.

Vijayakumar. L, Satheesh-Babu. R, "Does 'no pesticide' reduce suicides?” Int J Soc Psychiatry, 55:401-6, 2009.

Vijayakumar. L, Umamaheswari. C, Shujaath Ali .Z.S, Devaraj. P, Kesavan. K, Intervention for suicide attempters: A randomized controlled study, Indian J Psychiatry, 53: 244-8, 2011.

Waern M, Rubenowitz E, Runeson B, Skoog I, Wilhelmson K, Allebeck P. Burden of illness and suicide in elderly people: Case-control study. BMJ; 324:1355-1357, 2002. 
World Health Organization. Distribution of Suicide Rates $(100,000)$ by Gender and Age, Geneva: Author, Available from: http://www.who.int/mental_health/prevention/suicide_rates_charte /en/index.html. 